\title{
Serotyping, Antimicrobial Resistance Profile and Virulence Genes of Salmonella Serovars Isolated from Human, Animals and Birds
}

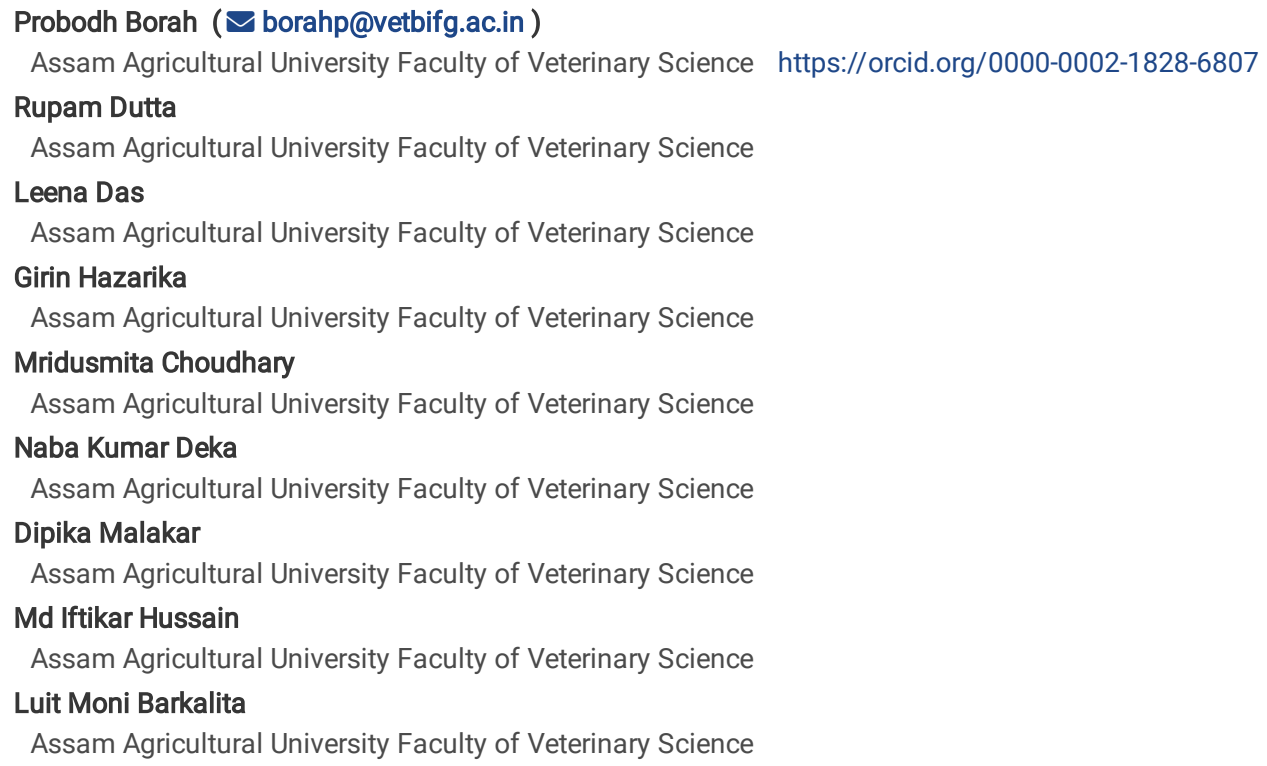

Keywords: Salmonella, prevalence, antimicrobial resistance, virulence gene.

Posted Date: July 14th, 2021

DOl: https://doi.org/10.21203/rs.3.rs-681026/v1

License: (c) (i) This work is licensed under a Creative Commons Attribution 4.0 International License. Read Full License 


\section{Abstract}

The study was undertaken to investigate the prevalence, antimicrobial susceptibility, antimicrobial resistance and virulence genes of Salmonella isolates recovered from human and different species of animals and birds. Out of 88 (7.15\%), 21 (23.86\%) belonged to Salmonella enterica subsp. enterica serovar Weltevreden, $22(25 \%)$ to serovar Enteritidis, 16 (18.2\%) to serovar Typhi and 14 (15.9\%) to serovar Newport, while 7 (7.95\%) isolates were found to be untypable. Among the 88 isolates, $45.45 \%$ showed resistance to ampicillin, $61.36 \%$ to tetracycline, $61.18 \%$ to cefotaxime, $65.90 \%$ to gentamicin, $48.86 \%$ to trimethoprim, $11.36 \%$ to ceftriaxone, $10.22 \%$ to chloramphenicol, and $7.95 \%$ each to ciprofloxacin and cefepime. Most of the isolates were susceptible to a low MIC $(\leq 0.25 \mu \mathrm{g} / \mathrm{ml})$ of Cefepime, Cefotaxime, Ciprofloxacin, Ceftriaxone and Co-trimoxazole and a moderate MIC $(0.5 \mu \mathrm{g} / \mathrm{ml}-4 \mu \mathrm{g} / \mathrm{ml})$ of Ampicillin, Tetracycline, Gentamicin and Chloramphenicol. The resistance genes, blaTEM, tetA and $d f r A 12$ were most prevalent, irrespective of the host of origin of the isolates. While invA was used for molecular detection of Salmonella, other virulence genes, viz. sipA, sipB, sipC, stn and T2544 were also detected in all (100\%) the Salmonella isolates. Total $69.32 \%$ of tested samples were found to be contaminated with multi-drug resistant (MDR) Salmonella and various virulence genes were present among the isolated serovars. Another virulence-associated gene, T2544 (pagN) could also be found in all the isolates, irrespective of serovar or host of origin suggesting the possibility of using this gene as a marker for identification of pathogenic Salmonella isolates. This study highlights the importance of continuous monitoring and surveillance for pathogenic salmonellae and their potential risks to both human and animal.

\section{Introduction}

Bacteria under the genus Salmonella are found in the intestinal tract of many animals, including cattle, pigs, horses, other mammals, reptiles, amphibians, and poultry (e.g., chickens, ducks, geese, and turkeys) are capable of causing infections in these species (Hale et al. 2012). Salmonella infection in human typically manifests as acute gastroenteritis that develops 12-72 hours after exposure. Young children, persons $>65$ years of age, and immuno-compromised persons are at greater risk for serious complications, including septicemia, joint or brain infections, and death (Giannella et al. 1996). The genus Salmonella consists of two species, namely Salmonella bongori and Salmonella enterica (Issenhuth-Jeanjean et al. 2014). Salmonella bongori is classically called as the Salmonella of lizards and it is mostly found in reptiles causing diarrhoea. Salmonella enterica is predominantly found in human and animals causing severe diarrhoea and fever. The species $S$. enterica has six subspecies namely enterica, salamae, arizonae, houtonae, indica and diarizonae. All of these subspecies have different serotypes. So far, a total of 2659 serotypes of $S$. enterica have been reported. Out of that, $S$. enterica subsp. enterica has the highest 1586 serotypes (Issenhuth-Jeanjean et al. 2014). Although all serotypes must be considered as potential human pathogens, only a limited number of serotypes are attributed to be the cause of infection in humans and animals. Most of the enteric diseases in human and animals with severe diarrhoea and fever are caused by different serotypes of $S$. enetrica subsp. enterica. This subsp. is one of the leading causes of zoonotic food-borne disease worldwide (Voetsch et al. 2004). It is estimated that in 2019, there were 2,12,500 and 4,100 cases of infections due to drug resistant non-typhoidal Salmonella and drug resistant Salmonella Typhi, respectively only in USA (CDC report; 2019). Antimicrobial Resistance Surveillance and Research Network (AMRSN) of India has categorized Salmonella (typhoidal and non-typhoidal) into enteric fever pathogens and diarrhoeagenic bacterial organisms groups out of a total of six groups chosen by ICMR (Walia et al. 2019) for developing a comprehensive plan on Anti-microbial resistance (AMR) surveillance in India. Salmonella species, specifically those which are fluoroquinolone resistant are categorized as "high priority" by WHO (WHO Report, 2017). MDR Salmonella carrying several classes of virulence genes have been detected in duck meat in China (Chen et al. 2020). Outbreaks of S. Typhi recently occurred in Bangladesh (Tanmoy et al. 2018) and Pakistan (Klemm et al. 2018) with the presence of XDR strains resistant to Ceftriaxone and several other antibiotics. Salmonella Typhimurium showed a high frequency of occurrence in poultry $(41.40 \%)$ and humans (43\%), and $S$. Weltevreden was found to be of zoonotic significance in India and has been recorded as one of the five most frequently isolated serovars (Kumar et al. 2009). In Assam, isolation of $S$. Weltevreden, $S$. Choleraesuis, $S$. Paratyphi B and $S$. Typhimurium from pigs (Rajkhowa et. al 2018), and S. Enteritidis, S. Gallinarum, S. Typhimurium, S. Newport and S. Indiana from poultry (Rahman et al. 1997; Rajkhowa et. al 2018) have been reported.

Emergence and spread of antimicrobial resistance among zoonotic Salmonella has become a public health threat. Importantly, Salmonella strains having "clinically important resistance" to some agents like extended spectrum cephalosporins and fluoroquinolones have been isolated from livestock (Li et al. 2013). In most developing countries, misuse and overuse of antibiotics has contributed to the increasing trend of multi-resistance in Salmonella (Eddra et al. 2017). Salmonella with antibiotic resistance in contaminated products could infect humans directly or transmit their resistance genes to human pathogens through the food chain, leading to failure of antibiotic treatment and may pose a serious threat to human health. The aim of the current study was to investigate the prevalence, antimicrobial resistance and virulence gene profiles of Salmonella serovars isolated from human and different species of animals and birds.

\section{Materials And Methods}

\section{Collection of samples}

A total of 1231 different samples consisting of faecal swabs from diarrhoeic (83) and apparently healthy (60) human, apparently healthy (101) and diseased (165) cattle, diarrhoeic (60) and apparently healthy (30) pigs, diarrhoeic (53) and apparently healthy (50) goats, diarrhoeic (302) and apparently healthy (103) poultry, apparently healthy (208) wild birds, apparently healthy (8) Gecko gecko, apparently healthy (6) tigers and mice (2) were collected from different parts of Assam. Type of samples was either faecal matter or part of intestine, in case of dead animals. Fresh samples were collected in sterile sample containers or in Carry-Blair medium, in case of anticipated delay from collection to processing by not more than 48 hours.

\section{Isolation}

Immediately after receiving the samples in the laboratory, they were put in pre-enrichment non-selective medium (sterile buffered peptone water broth) and incubated at $37^{\circ} \mathrm{C}$ for overnight. It was followed by inoculation of $1 \mathrm{ml}$ overnight broth into $9 \mathrm{ml}$ of selective enrichment (Selenite broth or Rappaport 
Vassiliadis soy peptone) broth and incubated at $37^{\circ} \mathrm{C}$ for overnight. Both broth cultures were kept at constant shaking at 250 rpm in a shaking incubator. Overnight turbid broth was inoculated to Brilliant Green Agar (BGA) and suspected positive colonies were picked and streaked on MacConkey's Lactose Agar (MLA). Both plates were incubated overnight at $37^{\circ} \mathrm{C}$. For preliminary identification of Salmonella, the suspected cultures were subjected to biochemical tests, viz. indole, urease and $\mathrm{H}_{2} \mathrm{~S}$ production, as well as production of lysine and ornithine decarboxylase. Additionally, growth characteristics on Triple Sulphate Iron (TSI) agar slants were also studied for determination of K/A reaction.

\section{Molecular detection of Salmonella}

Reference strains of Salmonella used in this study (Table. 1) were obtained from Microbial Type Culture Centre (MTCC), Chandigarh, American Type Culture Centre, USA and National Institute of Cholera and Enteric Diseases, ICMR, (NICED), Kolkata. The isolates were grown in Luria Bertani (LB) broth for overnight at $37^{\circ} \mathrm{C}$ and $2 \mathrm{ml}$ of the overnight cultures were centrifuged at $10,000 \mathrm{~g}$ for 10 minutes. The supernatants were discarded and the pellets were suspended in a total volume of $100 \mu \mathrm{l}$ of $1 \mathrm{X}$ TE buffer $(10 \mathrm{mM}$ Tris- $\mathrm{HCl}, 1 \mathrm{mM}$ EDTA, $\mathrm{pH} 8.0)$ and boiled at $100^{\circ} \mathrm{C}$ for 10 minutes. After boiling, the cell suspensions were immediately cooled on ice for 10 minutes and centrifuged at $15,000 \mathrm{~g}$ for 10 minutes at $4^{\circ} \mathrm{C}$. The supernatant was then collected without disturbing the sediment. Isolated DNA was quantified in Nanodrop 1000 and stored in $-20^{\circ} \mathrm{C}$ for future use as template DNA for PCR (Choudhury et al. 2016).

The isolates were confirmed as Salmonella by detecting the specific gene invA by simplex PCR. The specific primer pairs used (Galan et al. 1992) for this purpose was

5'-ACCACGCTCTTTCGTCTGG-3/ and 5'GAACTGACTACGTAGACGCTC-3'. A reaction mixture of $25 \mu$ was prepared with $12.5 \mu$ of Dream Taq PCR master mix (Fermentus), $0.5 \mu \mathrm{l}$ each primer $(10 \mathrm{pmol} / \mu \mathrm{l}), 1.0 \mu \mathrm{l}$ of template DNA and $10.5 \mu \mathrm{l}$ of nuclease-free water. PCR condition was standardized at $94^{\circ} \mathrm{C}$ for 5 minutes followed by 30 cycles at $94^{\circ} \mathrm{C}$ for 30 seconds, at $56^{\circ} \mathrm{C}$ for 1 minute and at $72^{\circ} \mathrm{C}$ for 1 minute. Final extension was given at $72^{\circ} \mathrm{C}$ for 5 minutes followed by infinite halt at $4^{\circ} \mathrm{C}$. The amplified products were run on $1.5 \%$ agarose gel, and visualized and documented in Gel Doc XR ${ }^{+}(\mathrm{Bio}-\mathrm{Rad})$.

\section{Serotyping}

The Salmonella isolates were sent for serotyping at National Institute of Cholera and Enteric Diseases (NICED), Kolkata, India, and National Salmonella and Escherichia Centre (NSEC), Central Research Institute, Kasauli, Himachal Pradesh, India.

\section{Antibiotic Susceptibility Testing and MIC}

Antibiotic sensitivity and MIC values were determined by disc dilution method and E-test using $6 \mathrm{~mm}$ antibiotic disc (HiMedia) and Ezy MIC ${ }^{\mathrm{TM}}$ (HiMedia) strips, respectively, according to Clinical and Laboratory Standards Institute guideline (CLSI Guideline, 2017) [19]. Pure colonies from agar plate were inoculated in Luria Bertani broth for 6-8 hours to get log phase growth. Following incubation, the turbidity was adjusted to match that of a $0.5 \mathrm{McFarland}$ standard. The container of discs and strips were removed from the $-20^{\circ} \mathrm{C}$ to equilibrate to room temperature for 30 minutes. Sterile $20 \% \mathrm{Muller}$ Hinton Agar (SRL) plates were inoculated by swabbing with sterile cotton swab (HiMedia) evenly. Antibacterial discs and Ezy MIC ${ }^{\mathrm{TM}}$ strips were placed on the surface aseptically as per manufacturer's instructions and kept for incubation at $37^{\circ} \mathrm{C}$ for overnight. Zone of inhibition and breakpoint values were recorded after the overnight incubation. A total of nine antimicrobial agents were used for both the tests. Ampicillin (AMP), Tetracycline (TET), Cefotaxime (CTX), Ciprofloxacin (CIP), Gentamicin (GEN), Chloramphenicol (CHL), Cefepime (CPM) and Ceftriaxone (CTR) were common for both, whereas Trimethoprim (TR) used in disc diffusion method was replaced by Co-trimoxazole (COT) in E-test (Table. 2).

\section{Resistance and virulence gene screening}

Salmonella isolates exhibiting resistance to different antimicrobial agents were then subjected to PCR for detecting resistance genes of concerned antibiotics. Resistance genes blaOXA, bla ${ }^{T E M}$, blaPSE ${ }^{-1}, \operatorname{dfr} A 1, \operatorname{dfr} A 12$, tet( $(A)$, tet( $\left.B\right)$ and tet(G) were detected using reported primers and PCR conditions (Table. 3). PCR reaction mixture constituents were same as mentioned earlier for invA gene detection. All the Salmonella isolates were screened for the presence of a total of 11 virulence genes including invA universally present in all isolates. The primer sets used for PCR amplification of $\operatorname{sip} A, \operatorname{sip} B$, $\operatorname{sip} C$, stn, $\operatorname{sop} B$, sopE, pefA, sefC, fepA and pagNalong with PCR conditions are listed in Table. 4. PCR reaction mixture constituents were same as mentioned earlier. The amplified products were run on $1.5 \%$ agarose gel and visualized under Gel Doc XR ${ }^{+}$(Bio-Rad, USA).

\section{Results}

\section{Isolation and identification}

Out of 1231 samples examined, Salmonella was recovered from 88 (7.15\%). Among the 663 clinical samples collected from man and animals with a history of diarrhoea, $68(10.26 \%)$ were positive for Salmonella, while out of 568 samples collected from apparently healthy animals and birds, 20 ( $3.52 \%)$ were positive for Salmonella (Table 5). All the 88 Salmonella isolates recovered from different sources fermented glucose, mannitol and dulcitol, but did not ferment lactose and sucrose. All the isolates were positive for methyl red test, citrate utilization and hydrogen sulphide production but were negative for indole production, Voges-Proskauer (VP) test and urease production. The isolates showed yellow butt, black middle and pink top on inoculation into Triple Sugar Iron (TSI) Agar slant. 
Table 1

List of Reference strains of Salmonella serotypes

\begin{tabular}{|lll|}
\hline SI no & Serotype name & Strain no \\
\hline 1 & Salmonella Typhimurium & MTCC 98 \\
\hline 3 & Salmonella Typhi & MTCC 8767 \\
\hline 4 & Salmonella Gallinarum & MTCC 2992 \\
\hline 5 & Salmonella Newport & MTCC 3229 \\
\hline 7 & Salmonella Virchow & MTCC 1166 \\
\hline 9 & Salmonella Enteritidis & ATCC 13076 \\
\hline 10 & Salmonella Vellore & NCTC 4840 \\
\hline 11 & Salmonella Abony & NCTC 6017 \\
\hline 12 & Salmonella Paratyphi A & NICED C6915 \\
\hline 13 & Salmonella Paratyphi B & NICED NK3727 \\
\hline
\end{tabular}

Table 2

Selected antimicrobial agents used for Antibiotic Susceptibility Testing and MIC

\begin{tabular}{|lllll|}
\hline SI. no. & Disc diffusion & \multicolumn{3}{c|}{ E- test } \\
\cline { 2 - 5 } & Antimicrobial agent & $\begin{array}{l}\text { Con. } \\
(\mu \mathrm{g})\end{array}$ & Antimicrobial agent & $\begin{array}{l}\text { Con. } \\
\text { gradient }(\mu \mathrm{g} / \mathrm{ml})\end{array}$ \\
\hline 1 & Ampicillin (AMP) & 10 & Ampicillin (AMP) & $0.016-256$ \\
\hline 2 & Tetracycline (TE) & 30 & Tetracycline (TET) & $0.016-256$ \\
\hline 3 & Cefotaxime (CTX) & 30 & Cefotaxime (CTX) & $0.016-256$ \\
\hline 5 & Ciprofloxacin (CIP) & 5 & Ciprofloxacin (CIP) & $0.002-32$ \\
\hline 6 & Gentamicin (GEN) & 30 & Gentamicin (GEN) & $0.016-256$ \\
\hline 7 & Chloramphenicol (C) & 30 & Chloramphenicol (CHL) & $0.016-256$ \\
\hline 8 & Cefepime (CPM) & 30 & Cefepime (CPM) & $0.016-256$ \\
\hline 9 & Trimethoprim (TR) & 5 & Co-trimoxazole (COT) & $0.002-32$ \\
\hline
\end{tabular}


Table 3

List of primers used in the study for detection of resistance genes in Salmonella

\begin{tabular}{|c|c|c|c|c|}
\hline R-genes & Primer sequence & Amplicon size (bp) & Annealing temp $\left({ }^{\circ} \mathrm{C}\right)$ & Reference \\
\hline \multirow[t]{2}{*}{ blaOXA (AMP) } & F-AGCAGCGCCAGTGCATCA & 708 & 60 & Guerra et al. (2001) \\
\hline & R-ATTCGACCCCAAGTTTCC & & & \\
\hline \multirow[t]{2}{*}{ blaTEM (AMP) } & F-TTGGGTGCACGAGTGGGT & 504 & 57 & Arlet and Phillippon (1991) \\
\hline & R-TAATTGTTGCCGGGAAGC & & & \\
\hline \multirow[t]{2}{*}{ blaPSE-1 (AMP) } & F-CGCTTCCCGTTAACAAGTAC & 420 & 58 & Sandvang et al. (1997) \\
\hline & R-CTGGTTCATTTCAGATAGCG & & & \\
\hline dfra1 & F- GTGAAACTATCACTAATGG & 470 & 50 & Guerra et al. (2001) \\
\hline$(\mathrm{TR})$ & R- CCCTTTTGCCAGATTTGG & & & \\
\hline \multirow[t]{2}{*}{ dfrA12 (TR) } & F- ACTCGGAATCAGTACGCA & 463 & 50 & Guerra et al. (2001) \\
\hline & R- GTGTACGGAATTACAGCT & & & \\
\hline \multirow[t]{2}{*}{$\operatorname{tet}(A)$} & F- GCTACATCCTGCTTGCCT & 210 & 57 & Ng et al. (1999) \\
\hline & R-CATAGATCGCCGTGAAGA & & & \\
\hline \multirow[t]{2}{*}{$\operatorname{tet}(B)$} & F-TTGGTTAGGGGCAAGTTTTG & 659 & 57 & $\mathrm{Ng}$ et al. (1999) \\
\hline & R-GTAATGGGCCAATAACACCG & & & \\
\hline \multirow[t]{2}{*}{$\operatorname{tet}(G)$} & F- GCTCGGTGGTATCTCTGC & 468 & 57 & $\mathrm{Ng}$ et al. (1999) \\
\hline & R- AGCAACAGAATCGGGAAC & & & \\
\hline
\end{tabular}

Table 4

List of primers used in the study for detection of virulence genes in Salmonella

\begin{tabular}{|c|c|c|c|}
\hline Target Gene & Primer sequence $\left(5^{\prime}-3^{\prime}\right)$ & $\begin{array}{l}\text { Primer concentration } \\
(\mathrm{pMol} / \mu \mathrm{l})\end{array}$ & Product size (bp) \\
\hline \multirow[t]{2}{*}{$\operatorname{sip} A$} & Forward - CGCAAATCATAAAAGATGGC & 20 & 646 \\
\hline & Reverse-CGGCTATTATCAATCGTCTT & & \\
\hline \multirow[t]{2}{*}{$\operatorname{sip} B$} & Forward -CTAACGTGCTGAAACAGTTG & 10 & 160 \\
\hline & Reverse-AACGCCACTTTATTTAGGGT & & \\
\hline \multirow[t]{2}{*}{$\operatorname{sip} C$} & Forward -TCTTAATGATGCGACGCTTA & 10 & 365 \\
\hline & Reverse-TGGTCGATTTACGTGAACTT & & \\
\hline \multirow[t]{2}{*}{ stn } & Forward -ATTGAGCGCTTTAATCTCCT & 10 & 543 \\
\hline & Reverse- GCTGTTGAATCTGTACCTGA & & \\
\hline \multirow[t]{2}{*}{$\operatorname{sop} B$} & Forward - GCATCTCTAAACGCTACTG & 10 & 470 \\
\hline & Reverse- GCTTCTATCACTCAGCTTCA & & \\
\hline \multirow[t]{2}{*}{ sopE } & Forward - GTAGGGCAGTATTAACCAG & 10 & 254 \\
\hline & Reverse- TTTATCTCCCTAGGTAGCCC & & \\
\hline \multirow[t]{2}{*}{ pefA } & Forward - CCAAAGTACTGGTTGAAAG & 20 & 185 \\
\hline & Reverse- TATTTGTAAGCCACTGCGAA & & \\
\hline \multirow[t]{2}{*}{ sefC } & Forward - GCAGGTCCAAAACTATACA & 10 & 609 \\
\hline & Reverse- CGATAACGAAACACCATTT & & \\
\hline \multirow[t]{2}{*}{ fepA } & Forward -CGACGTGTCAGAAATCATCC & 10 & 382 \\
\hline & Reverse- GCCTTCGTCTTTATGTTCCG & & \\
\hline \multirow[t]{2}{*}{$\operatorname{pagN}$} & Forward -GAATCAACTCAACCTTCAGC & 10 & 105 \\
\hline & Reverse-AGTCATAACCGATAGCAACC & & \\
\hline
\end{tabular}


Table 5

Isolation of Salmonella from human and different species of animals and birds

\begin{tabular}{|llllll|}
\hline \multirow{2}{*}{ Host } & \multicolumn{2}{l}{ No. of samples examined } & \multicolumn{2}{l|}{ No. of isolates recovered } \\
\cline { 2 - 6 } & Total & Diarnoeic & Apparently Healthy & Diarthoeic & Apparently Healthy \\
\hline Poultry & 405 & 302 & 103 & $17(5.63 \%)$ & $7(6.8 \%)$ \\
\hline Cattle & 266 & 165 & 101 & $18(10.91 \%)$ & - \\
\hline Wild Birds & 208 & - & 208 & - & $10(4.81 \%)$ \\
\hline Human & 143 & 83 & 60 & $28(33.73 \%)$ & - \\
\hline Goat & 103 & 53 & 50 & - & - \\
\hline Pig & 90 & 60 & 30 & $5(8.3 \%)$ & - \\
Gecko & 8 & - & 8 & - & $1(12.5 \%)$ \\
\hline Tiger & 6 & - & 6 & - & $1(16.67 \%)$ \\
\hline Mice & 2 & - & 2 & - & $1(50 \%)$ \\
Total & 1231 & 663 & 568 & $68(10.26 \%)$ & $20(3.52 \%)$ \\
\hline
\end{tabular}

\section{Serotyping of Salmonella}

Out of the 88 isolates, 21 (23.86\%) belonged to S. enterica subsp. enterica serovar Weltevreden, 22 (25\%) to serovar Enteritidis, 16 (18.20\%) to serovar Typhi and $14(15.90 \%)$ to serovar Newport, while 7 (7.95\%) isolates were found to be untypable. Detailed results of serotyping of the Salmonella strains are presented in (Table 6). In case of human samples, $S$. Typhi (62.50\%) was the most frequently isolated serovar, followed by serovars Weltevreden and Newport (Figure. 1). On the other hand, S. Newport (52.63\%) was found to be the most abundant serovar in cattle followed by serovars Weltevreden and Enteritidis (Figure. 2).

Table 6

Distribution of various serovars among Salmonella isolates recovered from different sources

\begin{tabular}{|lllll|}
\hline SI. No. & Serotype & Antigenic Structure & No. of isolates & Source/ Host \\
\hline 1 & Salmonella Enteritidis & $9,12: \mathrm{g}, \mathrm{m}$ & 22 & Cattle (2), Pig (1), Human (2), Poultry (13), Wild Birds (4) \\
\hline 2 & Salmonella Weltevreden & $3\{10\}, \mathrm{r}, \mathrm{Z}_{6}$ & 21 & Poultry (5), Human (4), Cattle (7), Pig (1), Wild Birds (3), Gecko gecko (1) \\
\hline 3 & Salmonella Typhi & $9,12, \mathrm{Vi, \textrm {d }}$ & 16 & Human \\
\hline 4 & Salmonella Newport & $6,8, \mathrm{e}, \mathrm{h}, 1,2$ & 14 & Cattle (9), Human (2), Poultry (3) \\
\hline 5 & Salmonella Typhimurium & $1,4,5,12, \mathrm{i}, 1,2$ & 2 & Human \\
\hline 6 & Salmonella Litchfield & $8,6,8, \mathrm{l}, \mathrm{v}, 1,2$ & 2 & Poultry \\
\hline 7 & Salmonella Kentucky & $8,8,20, \mathrm{l}, \mathrm{z} 6$ & 1 & Tiger \\
\hline 8 & Salmonella Idikan & $13,1,3,23, \mathrm{i}, 1,5$ & 1 & Human \\
\hline 9 & Salmonella Paratyphi B & $1,4,5,12, \mathrm{~b}, 1,2$ & 1 & Human \\
\hline 10 & Salmonella Virchow & $7,6,7,14, \mathrm{r}, 1,2$ & 1 & Mouse \\
\hline 11 & Untypable & & 7 & Poultry (1), Pig (3), Wild Birds (3) \\
\hline
\end{tabular}

\section{Antimicrobial Resistance Detected By Disc Diffusion Test}

All the 88 Salmonella isolates recovered from different sources in the present study were subjected to antimicrobial susceptibility test by disc diffusion method. Of the 88 isolates, 40 (45.45\%) showed resistance to ampicillin, $54(61.36 \%)$ to tetracycline, $60(61.18 \%)$ to cefotaxime, 58 (65.90\%) to gentamicin, 43 (48.86\%) to trimethoprim, $10(11.36 \%)$ to ceftriaxone, $9(10.22 \%)$ to chloramphenicol, and $7(7.95 \%)$ each to ciprofloxacin and cefepime. Of the 11 serovars, S. Weltevreden and $S$. Typhi and the untypable isolates showed resistance to all the antibiotics under test. Cefotaxime showed the highest resistance (61.18\%), whereas ciprofloxacin and cefepime showed the least resistance (7.95\%). Salmonella Virchow showed resistance to three antibiotics (Tetracycline, Gentamicin and Chloramphenicol), S. Kentucky to four (Ampicillin, Tetracycline, Cefotaxime and Gentamicin) and S. Paratyphi B to four (Tetracycline, Cefotaxime, Gentamicin and Trimethoprim) antimicrobial agents (Table 7). Ciprofloxacin resistance was shown by the isolates from poultry, wild bird, pig and human. Most of the $S$. Typhi isolates from human showed resistance to all the nine antibiotics tested. All the isolates from different hosts showed resistance to tetracycline except one $S$. Weltevreden isolate from Gecko gecko. None of the isolates of serovars $S$. Litchfield, S. Newport, $S$. Kentucky, S. Idikan, S. Paratyphi B and $S$. Virchow showed resistance to ciprofloxacin (Table. 8). 
Table 7

Resistance patterns of Salmonella isolates of different serovars from different sources against different antimicrobial agents

\begin{tabular}{|c|c|c|c|c|c|c|c|c|c|c|}
\hline Serovars & $\begin{array}{l}\text { No. of } \\
\text { isolates }\end{array}$ & $\begin{array}{l}\text { Ampicillin } \\
\text { (10mcg) }\end{array}$ & $\begin{array}{l}\text { Tetracycline } \\
\text { (30mcg) }\end{array}$ & $\begin{array}{l}\text { Cefotaxime } \\
\text { (30mcg) }\end{array}$ & $\begin{array}{l}\text { Ciprofloxacin } \\
(5 \mathrm{mcg})\end{array}$ & $\begin{array}{l}\text { Gentamicin } \\
(30 \mathrm{mcg})\end{array}$ & $\begin{array}{l}\text { Chloramphenicol } \\
(30 \mathrm{mcg})\end{array}$ & $\begin{array}{l}\text { Cefepime } \\
(30 \mathrm{mcg})\end{array}$ & $\begin{array}{l}\text { Ceftriaxone } \\
(30 \mathrm{mcg})\end{array}$ & $\begin{array}{l}\text { Trim } \\
(5 \mathrm{mc}\end{array}$ \\
\hline S. Litchfield & 2 & $1(50)$ & $1(50)$ & $2(100)$ & 0 & $2(100)$ & 0 & 0 & 0 & $1(5 \mathrm{C}$ \\
\hline $\begin{array}{l}\text { S. } \\
\text { Weltevreden }\end{array}$ & 21 & 7 (33.34) & $12(57.14)$ & $14(66.67)$ & $1(4.76)$ & $10(47.61)$ & $2(9.52)$ & $2(9.52)$ & $2(9.52)$ & $10(4$ \\
\hline S. Newport & 14 & $9(64.28)$ & $7(50)$ & $7(50)$ & 0 & 11 (78.57) & $3(21.42)$ & $2(14.28)$ & $3(21.42)$ & $5(35$ \\
\hline S.Typhi & 16 & $5(31.25)$ & $13(81.25)$ & $11(68.75)$ & $3(18.75)$ & $10(62.5)$ & $1(6.25)$ & $1(6.25)$ & $3(18.75)$ & $8(5 C$ \\
\hline$S$. Enteritidis & 22 & $\begin{array}{l}10 \\
(45.45)\end{array}$ & $12(54.55)$ & $15(68.18)$ & $1(4.54)$ & $14(63.64)$ & 0 & $1(4.54)$ & $1(4.54)$ & $13(5$ \\
\hline S. Kentucky & 1 & $1(100)$ & $1(100)$ & $1(100)$ & 0 & $1(100)$ & 0 & 0 & 0 & 0 \\
\hline $\begin{array}{l}\text { S. } \\
\text { Typhimurium }\end{array}$ & 2 & $1(50)$ & $2(100)$ & $1(50)$ & $1(50)$ & $1(50)$ & 0 & 0 & 0 & $1(5 \mathrm{C}$ \\
\hline S. Idikan & 1 & $1(100)$ & $1(100)$ & $1(100)$ & 0 & $1(100)$ & $1(100)$ & 0 & 0 & $1(1 \mathrm{C}$ \\
\hline $\begin{array}{l}\text { S. Paratyphi } \\
\text { B }\end{array}$ & 1 & 0 & $1(100)$ & $1(100)$ & 0 & $1(100)$ & 0 & 0 & 0 & $1(1 \mathrm{C}$ \\
\hline S. Virchow & 1 & 0 & $1(100)$ & 0 & 0 & $1(100)$ & $1(100)$ & 0 & 0 & 0 \\
\hline \multirow[t]{2}{*}{ Untypable } & 7 & $5(71.43)$ & $3(42.85)$ & $7(100)$ & $1(14.28)$ & $6(85.71)$ & $1(14.28)$ & $1(14.28)$ & $1(14.28)$ & $3(42$ \\
\hline & 88 & $\begin{array}{l}40 \\
(45.45)\end{array}$ & $54(61.36)$ & $60(61.18)$ & $7(7.95)$ & $58(65.9)$ & 9 (10.22) & $7(7.95)$ & $10(11.36)$ & $43(4$ \\
\hline
\end{tabular}


Table 8

Resistance patterns of Salmonella isolates from different host species against different antimicrobial agents

\begin{tabular}{|c|c|c|c|c|c|c|c|c|c|c|}
\hline Serovars & Host & $\begin{array}{l}\text { No. of } \\
\text { isolates }\end{array}$ & $\begin{array}{l}\text { Ampicillin } \\
\text { (10mcg) }\end{array}$ & $\begin{array}{l}\text { Tetracycline } \\
\text { (10mcg) }\end{array}$ & $\begin{array}{l}\text { Cefotaxime } \\
(30 \mathrm{mcg})\end{array}$ & $\begin{array}{l}\text { Ciprofloxacin } \\
(5 \mathrm{mcg})\end{array}$ & $\begin{array}{l}\text { Gentamicin } \\
(30 \mathrm{mcg})\end{array}$ & $\begin{array}{l}\text { Chloramphenicol } \\
(25 \mathrm{mcg})\end{array}$ & $\begin{array}{l}\text { Cefepime } \\
\text { (30mcg) }\end{array}$ & $\begin{array}{l}\text { Ceftriaxc } \\
(30 \mathrm{mcg})\end{array}$ \\
\hline \multirow[t]{2}{*}{ S. Litchfield } & Poultry & 2 & $1(50)$ & $1(50)$ & $2(100)$ & 0 & $2(100)$ & 0 & 0 & 0 \\
\hline & $\begin{array}{l}\text { Wild } \\
\text { bird }\end{array}$ & 3 & 1 (33.33) & 0 & $2(66.67)$ & 0 & $1(33.33)$ & 0 & 0 & 0 \\
\hline \multirow{5}{*}{$\begin{array}{l}S . \\
\text { Weltevreden }\end{array}$} & Poultry & 5 & $2(40)$ & $3(60)$ & $5(100)$ & $1(20)$ & $4(80)$ & $1(20)$ & 0 & $1(20)$ \\
\hline & Human & 4 & $1(28.57)$ & $4(100)$ & $4(100)$ & 0 & $2(50)$ & 0 & 0 & 0 \\
\hline & Cattle & 7 & $2(28.57)$ & $4(57.14)$ & $2(28.57)$ & 0 & 3 (42.85) & 0 & 1 (14.28) & $1(14.28)$ \\
\hline & Pig & 1 & 0 & $1(100)$ & 0 & 0 & 0 & $1(100)$ & $1(100)$ & 0 \\
\hline & Gecko & 1 & $1(100)$ & 0 & $1(100)$ & 0 & 0 & 0 & 0 & 0 \\
\hline \multirow[t]{3}{*}{ S. Newport } & Poultry & 3 & $1(33.33)$ & $1(33.33)$ & 1 (33.33) & 0 & $2(66.67)$ & $1(33.33)$ & 1 (33.33) & 1 (33.33) \\
\hline & Cattle & 9 & 7 (77.78) & $5(55.55)$ & $4(44.44)$ & 0 & 7 (77.78) & $1(11.11)$ & 0 & 0 \\
\hline & Human & 2 & $1(50)$ & $1(50)$ & $2(100)$ & 0 & $2(100)$ & $1(50)$ & $1(50)$ & $2(100)$ \\
\hline S. Typhi & Human & 16 & $5(31.25)$ & $13(81.25)$ & $11(68.75)$ & 3 (18.75) & $10(62.5)$ & $1(6.25)$ & $1(6.25)$ & $3(18.75)$ \\
\hline \multirow[t]{5}{*}{ S. Enteritidis } & $\begin{array}{l}\text { Wild } \\
\text { bird }\end{array}$ & 4 & $2(50)$ & $1(25)$ & $2(50)$ & $2(50)$ & 0 & 0 & $1(25)$ & $1(25)$ \\
\hline & Poultry & 14 & $8(57.14)$ & $7(50)$ & $9(64.28)$ & $1(7.14)$ & 8 (57.14) & 0 & 0 & 0 \\
\hline & Cattle & 2 & 0 & $2(100)$ & $2(100)$ & 0 & $2(100)$ & 0 & 0 & 0 \\
\hline & Pig & 1 & 0 & $1(100)$ & $1(100)$ & 0 & $1(100)$ & 0 & 0 & 0 \\
\hline & Human & 1 & 0 & $1(100)$ & $1(100)$ & 0 & 0 & 0 & 0 & 0 \\
\hline S. Kentucky & Tiger & 1 & $1(100)$ & $1(100)$ & $1(100)$ & 0 & $1(100)$ & 0 & 0 & 0 \\
\hline $\begin{array}{l}S . \\
\text { Typhimurium }\end{array}$ & Human & 2 & $1(50)$ & $2(100)$ & $1(50)$ & $1(50)$ & $1(50)$ & 0 & 0 & 0 \\
\hline S. Idikan & Human & 1 & $1(100)$ & $1(100)$ & $1(100)$ & 0 & $1(100)$ & $1(100)$ & 0 & 0 \\
\hline $\begin{array}{l}\text { S. Paratyphi } \\
\text { B }\end{array}$ & Human & 1 & 0 & $1(100)$ & $1(100)$ & 0 & $1(100)$ & 0 & 0 & 0 \\
\hline S. Virchow & Mouse & 1 & 0 & $1(100)$ & 0 & 0 & $1(100)$ & $1(100)$ & 0 & 0 \\
\hline \multirow[t]{3}{*}{ Untypable } & Poultry & 1 & $1(100)$ & $1(100)$ & $1(100)$ & 0 & $1(100)$ & 0 & 0 & 0 \\
\hline & Pig & 3 & 1 (33.33) & $1(33.33)$ & $3(100)$ & $3(100)$ & $2(66.67)$ & 1 (33.33) & 0 & 1 (33.33) \\
\hline & $\begin{array}{l}\text { Wild } \\
\text { bird }\end{array}$ & 3 & $3(100)$ & 1 (33.33) & $3(100)$ & 1 (33.33) & $3(100)$ & 0 & 1 (33.33) & 1 (33.33) \\
\hline
\end{tabular}

A total of $61(69.32 \%)$ isolates were found to be multi-drug resistant (MDR) and one among these isolates showed resistance to four antimicrobial agents (Cefotaxim, Tetracycline, Gentamicin and Ampicillin). Salmonella Weltevreden isolates showed resistance to Cefotaxime (92.31\%) and Tetracycline (69.23\%). Among the human isolates, $100 \%$ resistance was observed against Cefotaxime, Trimethoprim and Tetracycline. However, no resistance was shown against Ceftriaxone and Cefepime. Among the poultry isolates, 100\% resistance was observed against Cefotaxime, while no resistance was shown against Cefepime. Salmonella Newport isolates showed $100 \%$ resistance against Gentamicin and $77.78 \%$ resistance against both Ampicillin and Cefotaxime. Among the cattle isolates, Gentamicin and Ampicillin resistance was found to be $100 \%$, Cefotaxime resistance was $80 \%$ with no resistance to Ceftriaxone. Among S. Enteritidis isolates, Cefotaxime, Trimethoprim and Ampicillin resistance was found to be $100 \%, 92.86 \%$ and $35.71 \%$, respectively. Among the poultry isolates, Ampicillin, Cefotaxime and Tetracycline resistance was found to be $50 \%, 100 \%$ and $60 \%$, respectively. In $S$. Typhi, which is a human host-specific serovar, $83.33 \%$ resistance was found against Cefotaxime, Gentamicin and Tetracycline each, while resistance to Chloramphenicol and Ceftriaxone were found to be $8.33 \%$ and $25 \%$, respectively.

\section{Mic Determined By E-Test}

Most of the isolates had a MIC value of $\leq 0.125 \mu \mathrm{g} / \mathrm{ml}$ for Cefepime, Cefotaxim, Ciprofloxacin, Ceftriaxone and Co-trimoxazole followed by MIC value of 0.25 $\mu \mathrm{g} / \mathrm{ml}$ for Ampicillin and Tetracycline. The MIC values of $S$. Newport for all antimicrobial agents were found between $1 \mu \mathrm{g} / \mathrm{ml}$ and $16 \mu \mathrm{g} / \mathrm{ml}, \mathrm{for} S$. Typhi the values were between $0.5 \mu \mathrm{g} / \mathrm{ml}$ and $16 \mu \mathrm{g} / \mathrm{ml}$, for $S$. Weltevreden between $0.25 \mu \mathrm{g} / \mathrm{ml}$ and $16 \mu \mathrm{g} / \mathrm{ml}$ with only three isolates showing MIC values greater than $256 \mu \mathrm{g} / \mathrm{ml}$. For $S$. Enteritidis, Ampicillin and Tetracycline MIC values were mostly found between $0.5 \mu \mathrm{g} / \mathrm{ml}$ and $8 \mu \mathrm{g} / \mathrm{ml}$ with three isolates showing MIC values greater than $256 \mu \mathrm{g} / \mathrm{ml}$. Chloramphenicol and Gentamicin MIC values for $S$. Newport, $S$. Weltevreden, and $S$. Enteritidis were between $0.5 \mu \mathrm{g} / \mathrm{ml}$ and 4 
$\mu \mathrm{g} / \mathrm{ml}$, whereas the corresponding values were between $0.5 \mu \mathrm{g} / \mathrm{ml}$ and $8 \mu \mathrm{g} / \mathrm{ml}$ for $S$. Typhi isolates (Figures. 3). For $S$. Typhimurium, the MIC values were found to be between $\leq 0.125 \mu \mathrm{g} / \mathrm{ml}$ and $4 \mu \mathrm{l} / \mathrm{ml}$ for Cefepime, Cefotaxim, Ciprofloxacin, Ceftriaxone and Co-trimoxazole, while for the rest of the drugs, it varied from $4 \mu \mathrm{l} / \mathrm{ml}$ to $32 \mu \mathrm{l} / \mathrm{ml}$ (Table 9 ).

Table 9

Antimicrobial resistance phenotypes of Salmonella isolates $(n=88)$.

\begin{tabular}{|c|c|c|c|c|c|c|c|c|c|c|c|c|}
\hline \multirow[t]{2}{*}{ Antibiotics } & \multicolumn{12}{|c|}{ Minimum Inhibitory Concentration (MIC) Distribution $(\mu \mathrm{g} / \mathrm{ml})$ of 88 Salmonella isolates } \\
\hline & $\leq 0.125$ & 0.25 & 0.5 & 1 & 2 & 4 & 8 & 16 & 32 & 64 & 128 & $\geq 256$ \\
\hline AMP & 0 & 1 & 5 & 23 & 27 & 7 & 6 & 5 & 2 & 1 & 0 & 11 \\
\hline TET & 0 & 0 & 23 & 33 & 12 & 4 & 7 & 4 & 2 & 1 & 0 & 2 \\
\hline CPM & 64 & 15 & 0 & 3 & 1 & 0 & 1 & 0 & 0 & 0 & 0 & 4 \\
\hline CTX & 65 & 19 & 0 & 0 & 0 & 0 & 0 & 0 & 0 & 2 & 1 & 1 \\
\hline CIP & 65 & 12 & 1 & 0 & 0 & 2 & 2 & 1 & 5 & 0 & 0 & 0 \\
\hline $\mathrm{CHL}$ & 0 & 0 & 1 & 1 & 49 & 36 & 1 & 0 & 0 & 0 & 0 & 0 \\
\hline GEN & 1 & 2 & 22 & 51 & 6 & 1 & 5 & 0 & 0 & 0 & 0 & 0 \\
\hline CTR & 83 & 0 & 1 & 0 & 0 & 0 & 0 & 0 & 4 & 0 & 0 & 0 \\
\hline СОT & 85 & 3 & 0 & 0 & 0 & 0 & 0 & 0 & 0 & 0 & 0 & 0 \\
\hline
\end{tabular}

\section{Detection Of Resistance Genes}

Salmonella isolates showing resistance to Ampicillin, Tetracycline and Trimethoprim were further subjected to PCR for detection of resistance genes (blaTEM, blaPSE and blaOXA; tetA, tetB and tetG; drfA1, drfA12) corresponding to their phenotypic resistance patterns. Out of 41 isolates showing resistance to ampicillin, 2 (4.88\%) showed presence of blaOXA gene, 1 (2.44\%) showed blaPSE and 28 (63.64\%) showed BlaTEM gene. Only two isolates of serovar S. Enteritidis from poultry showed presence of blaOXA and one isolate of $S$. Typhi from human showed presence of blaPSE. The two $S$. Newport isolates from human and poultry did not possess blaTEM gene. Similarly, three $S$. Weltevreden isolates from poultry and one isolate each from wild birds and Gecko gecko belonging to the same serovar did not show presence of blaTEM gene. All the isolates (100\%) of $S$. Litchfield, $S$. Typhi, $S$. Typhimurium, $S$. Kentucky and $S$. Idikan as well as the untypable isolates showed presence of blaTEM gene.

Out of 54 isolates showing resistance to tetracycline, 9 (16.67\%) showed presence of tetA gene and 1 (1.85\%) showed presence of tetG gene, whereas none showed presence of tetB gene. Only one untypable isolate from poultry showed presence of tet $G$ gene. However, none of the isolates of $S$. Newport, $S$. Kentucky, $S$. Idikan, $S$. Paratyphi B and $S$. Virchow showed presence of tetA gene. Out of the 44 isolates showing resistance to trimethoprim, 5 (11.36\%) showed presence of resistance gene drfA1 but none showed presence of $d r f A 12$. The drfA1 gene was detected in two isolates each of $S$. Typhi and $S$. Enteritidis, and one isolate of $S$. Typhimurium (Table 10). 
Table 10

Screening of Salmonella isolates from different sources for resistance genes against different antimicrobial agents

\begin{tabular}{|c|c|c|c|c|c|c|c|c|c|c|c|c|}
\hline \multirow[t]{2}{*}{ Serovars } & \multirow{2}{*}{$\begin{array}{l}\text { No. of } \\
\text { isolates } \\
\text { showing } \\
\text { resistance }\end{array}$} & \multicolumn{3}{|c|}{$\begin{array}{l}\text { No. of isolates showing } \\
\text { positive for ampicillin } \\
\text { resistant genes }\end{array}$} & \multirow[t]{2}{*}{ Serovars } & \multirow{2}{*}{$\begin{array}{l}\text { No. of } \\
\text { isolates } \\
\text { showing } \\
\text { resistance }\end{array}$} & \multicolumn{3}{|c|}{$\begin{array}{l}\text { No. of isolates showing } \\
\text { positive for tetracycline } \\
\text { resistance genes }\end{array}$} & \multirow[t]{2}{*}{ Serovars } & \multirow{2}{*}{$\begin{array}{l}\text { No. of } \\
\text { isolates } \\
\text { showing } \\
\text { resistance }\end{array}$} & \multirow{2}{*}{$\begin{array}{l}\text { No. } \\
\text { trin } \\
\text { resi } \\
\text { gen } \\
\text { dfr/ }\end{array}$} \\
\hline & & blaOXA & blaPSE & blaTEM & & & tetA & tetB & tetG & & & \\
\hline S. Litchfield & 1 & 0 & 0 & $1(100)$ & S. Litchfield & 1 & $1(100)$ & 0 & 0 & $\begin{array}{l}\text { S. } \\
\text { Weltevreden }\end{array}$ & 11 & 0 \\
\hline S.Newport & 9 & 0 & 0 & $\begin{array}{l}7 \\
(77.78)\end{array}$ & S. Newport & 7 & 0 & 0 & 0 & S. Newport & 5 & 0 \\
\hline$S$. Enteritidis & 11 & $\begin{array}{l}2 \\
(18.18)\end{array}$ & 0 & $\begin{array}{l}6 \\
(54.54)\end{array}$ & S. Enteritidis & 12 & $\begin{array}{l}2 \\
(16.67)\end{array}$ & 0 & 0 & S. Enteritidis & 13 & 2 \\
\hline S.Typhi & 4 & 0 & $1(25)$ & $4(100)$ & S.Typhi & 13 & $\begin{array}{l}1 \\
(7.69)\end{array}$ & 0 & 0 & S. Typhi & 8 & 2 \\
\hline $\begin{array}{l}S . \\
\text { Weltevreden }\end{array}$ & 8 & 0 & 0 & $2(25)$ & $\begin{array}{l}S . \\
\text { Weltevreden }\end{array}$ & 12 & $\begin{array}{l}2 \\
(16.67)\end{array}$ & 0 & 0 & $\begin{array}{l}\text { S. } \\
\text { Typhimurium }\end{array}$ & 1 & 1 \\
\hline Untypable & 5 & 0 & 0 & $5(100)$ & Untypable & 3 & $\begin{array}{l}2 \\
(16.67)\end{array}$ & 0 & $\begin{array}{l}1 \\
(33.33)\end{array}$ & $\begin{array}{l}\text { S. Paratyphi } \\
\text { B }\end{array}$ & 1 & 0 \\
\hline S. Kentucky & 1 & 0 & 0 & $1(100)$ & S. Kentucky & 1 & 0 & 0 & 0 & S. Idikan & 1 & 0 \\
\hline $\begin{array}{l}S . \\
\text { Typhimurium }\end{array}$ & 1 & 0 & 0 & $1(100)$ & $\begin{array}{l}S . \\
\text { Typhimurium }\end{array}$ & 2 & $1(50)$ & 0 & 0 & S. Litchfield & 1 & 0 \\
\hline \multirow[t]{4}{*}{ S. Idikan } & 1 & 0 & 0 & $1(100)$ & S. Idikan & 1 & 0 & 0 & 0 & Untypable & 3 & 0 \\
\hline & & & & & $\begin{array}{l}\text { S. Paratyphi } \\
\text { B }\end{array}$ & 1 & 0 & 0 & 0 & & & \\
\hline & & & & & S. Virchow & 1 & 0 & 0 & 0 & & & \\
\hline & 41 & $\begin{array}{l}2 \\
(4.88)\end{array}$ & $\begin{array}{l}1 \\
(2.44)\end{array}$ & $\begin{array}{l}28 \\
(63.64)\end{array}$ & & 54 & $\begin{array}{l}9 \\
(16.67)\end{array}$ & 0 & $\begin{array}{l}1 \\
(1.85)\end{array}$ & & 44 & 5 \\
\hline
\end{tabular}

\section{Virulence Gene Detection}

All the 88 Salmonella isolates were subjected to simplex PCR for detection of 11 important virulence genes (Table 11). The different serovars of Salmonella showed varability in their virulence gene profiles. While invA was used as the internal control for molecular detection of Salmonella, virulence genes sipA, sipB, sipC, stn and T2544 were also detected in all (100\%) the Salmonella isolates, while fepA gene was present in 57 (64.77\%) isolates belonging to serovars Enteritidis (12), Weltervreden (14), Typhi (14), Newport (8), Litchfield and Idikan (one isolate each), and Typhimurium (2) and the untypable (5) isolates. The rest four virulence genes $\operatorname{sop} B(86.36 \%)$, sopE $(62.5 \%)$, pef $A(79.54 \%)$ and $\operatorname{sef} C(51.14 \%)$ were found to be present in varying percentage among the Salmonella serovars. Maximum numbers (5) of the 17 isolates carrying all the eleven genes under study were recovered from wild birds and human. Out of all the 88 isolates screened, a total of 11 (12.5\%) isolates belonging to serovars Weltervreden (7) and Typhi (4) were found to be positive for all eleven genes, while three other untypable isolates also carried all eleven genes. 
Table 11

Distribution of virulence genes in Salmonella isolates of different serovars isolated from various sources

\begin{tabular}{|c|c|c|c|c|c|c|c|c|c|c|c|c|}
\hline \multirow[t]{2}{*}{ Strains } & \multirow{2}{*}{$\begin{array}{l}\text { No of } \\
\text { isolates }\end{array}$} & \multicolumn{11}{|l|}{ Genes } \\
\hline & & invA & stn & $\mathrm{t} 2544$ & fepA & pefA & sopE & sopB & sefC & sipA & sip $B$ & sipc \\
\hline S. Litchfield & 2 & $\begin{array}{l}2 \\
(100 \%)\end{array}$ & $\begin{array}{l}2 \\
(100 \%)\end{array}$ & $\begin{array}{l}2 \\
(100 \%)\end{array}$ & $1(50 \%)$ & $1(50 \%)$ & 0 & $1(50 \%)$ & 0 & $\begin{array}{l}2 \\
(100 \%)\end{array}$ & $\begin{array}{l}2 \\
(100 \%)\end{array}$ & $\begin{array}{l}2 \\
(100 \%)\end{array}$ \\
\hline S. & 21 & $\begin{array}{l}21 \\
(100 \%)\end{array}$ & $\begin{array}{l}21 \\
(100 \%)\end{array}$ & $\begin{array}{l}21 \\
(100 \%)\end{array}$ & $\begin{array}{l}14 \\
(66.67 \%)\end{array}$ & $\begin{array}{l}18 \\
(85.7 \%)\end{array}$ & $\begin{array}{l}12 \\
(57.1 \%)\end{array}$ & $\begin{array}{l}17 \\
(80.95 \%)\end{array}$ & $\begin{array}{l}12 \\
(57.1 \%)\end{array}$ & $\begin{array}{l}21 \\
(100 \%)\end{array}$ & $\begin{array}{l}21 \\
(100 \%)\end{array}$ & $\begin{array}{l}21 \\
(100 \%)\end{array}$ \\
\hline S. Newport & 14 & $\begin{array}{l}14 \\
(100 \%)\end{array}$ & $\begin{array}{l}14 \\
(100 \%)\end{array}$ & $\begin{array}{l}14 \\
(100 \%)\end{array}$ & $\begin{array}{l}8 \\
(57.14 \%)\end{array}$ & $\begin{array}{l}13 \\
(92.86 \%)\end{array}$ & $\begin{array}{l}11 \\
(78.57 \%)\end{array}$ & $14(100 \%)$ & $\begin{array}{l}5 \\
(35.71 \%)\end{array}$ & $\begin{array}{l}14 \\
(100 \%)\end{array}$ & $\begin{array}{l}14 \\
(100 \%)\end{array}$ & $\begin{array}{l}14 \\
(100 \%)\end{array}$ \\
\hline S. Typhi & 16 & $\begin{array}{l}16 \\
(100 \%)\end{array}$ & $\begin{array}{l}16 \\
(100 \%)\end{array}$ & $\begin{array}{l}16 \\
(100 \%)\end{array}$ & $\begin{array}{l}14 \\
(87.5 \%)\end{array}$ & $\begin{array}{l}13 \\
(81.25 \%)\end{array}$ & $\begin{array}{l}13 \\
(81.25 \%)\end{array}$ & $\begin{array}{l}14 \\
(87.5 \%)\end{array}$ & $\begin{array}{l}6 \\
(37.5 \%)\end{array}$ & $\begin{array}{l}16 \\
(100 \%)\end{array}$ & $\begin{array}{l}16 \\
(100 \%)\end{array}$ & $\begin{array}{l}16 \\
(100 \%)\end{array}$ \\
\hline S. Enteritidis & 22 & $\begin{array}{l}22 \\
(100 \%)\end{array}$ & $\begin{array}{l}22 \\
(100 \%)\end{array}$ & $\begin{array}{l}22 \\
(100 \%)\end{array}$ & $\begin{array}{l}12 \\
(54.55 \%)\end{array}$ & $\begin{array}{l}14 \\
(63.64 \%)\end{array}$ & $11(50 \%)$ & $20(90.9 \%)$ & $\begin{array}{l}15 \\
(68.18 \%)\end{array}$ & $\begin{array}{l}22 \\
(100 \%)\end{array}$ & $\begin{array}{l}22 \\
(100 \%)\end{array}$ & $\begin{array}{l}22 \\
(100 \%)\end{array}$ \\
\hline S. Kentucky & 1 & $\begin{array}{l}1 \\
(100 \%)\end{array}$ & $\begin{array}{l}1 \\
(100 \%)\end{array}$ & $\begin{array}{l}1 \\
(100 \%)\end{array}$ & 0 & $1(100 \%)$ & $1(100 \%)$ & $1(100 \%)$ & 0 & $\begin{array}{l}1 \\
(100 \%)\end{array}$ & $\begin{array}{l}1 \\
(100 \%)\end{array}$ & $\begin{array}{l}1 \\
(100 \%)\end{array}$ \\
\hline $\begin{array}{l}\text { S. } \\
\text { Typhimurium }\end{array}$ & 2 & 2 & $\begin{array}{l}2 \\
(100 \%)\end{array}$ & $\begin{array}{l}2 \\
(100 \%)\end{array}$ & $2(100 \%)$ & $2(100 \%)$ & $1(50 \%)$ & $2(100 \%)$ & $1(50 \%)$ & $\begin{array}{l}2 \\
(100 \%)\end{array}$ & $\begin{array}{l}2 \\
(100 \%)\end{array}$ & $\begin{array}{l}2 \\
(100 \%)\end{array}$ \\
\hline S. Idikan & 1 & $\begin{array}{l}1 \\
(100 \%)\end{array}$ & $\begin{array}{l}1 \\
(100 \%)\end{array}$ & $\begin{array}{l}1 \\
(100 \%)\end{array}$ & $1(100 \%)$ & $1(100 \%)$ & 0 & $1(100 \%)$ & $1(100 \%)$ & $\begin{array}{l}1 \\
(100 \%)\end{array}$ & $\begin{array}{l}1 \\
(100 \%)\end{array}$ & $\begin{array}{l}1 \\
(100 \%)\end{array}$ \\
\hline $\begin{array}{l}\text { S. Paratyphi } \\
\text { B }\end{array}$ & 1 & $\begin{array}{l}1 \\
(100 \%)\end{array}$ & $\begin{array}{l}1 \\
(100 \%)\end{array}$ & $\begin{array}{l}1 \\
(100 \%)\end{array}$ & 0 & $1(100 \%)$ & $1(100 \%)$ & 0 & 0 & $\begin{array}{l}1 \\
(100 \%)\end{array}$ & $\begin{array}{l}1 \\
(100 \%)\end{array}$ & $\begin{array}{l}1 \\
(100 \%)\end{array}$ \\
\hline S. Virchow & 1 & $\begin{array}{l}1 \\
(100 \%)\end{array}$ & $\begin{array}{l}1 \\
(100 \%)\end{array}$ & $\begin{array}{l}1 \\
(100 \%)\end{array}$ & 0 & 0 & 0 & 0 & 0 & $\begin{array}{l}1 \\
(100 \%)\end{array}$ & $\begin{array}{l}1 \\
(100 \%)\end{array}$ & $\begin{array}{l}1 \\
(100 \%)\end{array}$ \\
\hline Untypable & 7 & $\begin{array}{l}7 \\
(100 \%)\end{array}$ & $\begin{array}{l}7 \\
(100 \%)\end{array}$ & $\begin{array}{l}7 \\
(100 \%)\end{array}$ & $\begin{array}{l}5 \\
(71.43 \%)\end{array}$ & $\begin{array}{l}6 \\
(85.71 \%)\end{array}$ & $\begin{array}{l}5 \\
(71.43 \%)\end{array}$ & $\begin{array}{l}6 \\
(85.71 \%)\end{array}$ & $\begin{array}{l}5 \\
(71.43 \%)\end{array}$ & $\begin{array}{l}7 \\
(100 \%)\end{array}$ & $\begin{array}{l}7 \\
(100 \%)\end{array}$ & $\begin{array}{l}7 \\
(100 \%)\end{array}$ \\
\hline \multirow[t]{2}{*}{ Total } & 88 & 88 & 88 & 88 & 57 & 70 & 55 & 76 & 45 & 88 & 88 & 88 \\
\hline & & $(100 \%)$ & $(100 \%)$ & $(100 \%)$ & (64.77\%) & (79.54\%) & $(62.5 \%)$ & $(86.36 \%)$ & (51.14\%) & $(100 \%)$ & $(100 \%)$ & $(100 \%)$ \\
\hline
\end{tabular}

The distribution of virulence genes according to the source of recovery of the isolates revealed that invA, $\operatorname{sip} A, \operatorname{sip} B, \operatorname{sip} C, \operatorname{stn}$ and $T 2544$ genes were present in all the isolates irrespective of their source of origin. The sefC gene is present in $45(51.14 \%)$ isolates from human, cattle, wild bird, pig, tiger and poultry. The genes sopB and sopE were present in 76 (86.36\%) and 55 (62.50\%) isolates, respectively, while pef $A$, sefC and fepA genes were present in 70 (79.54\%), 45 $(51.14 \%)$ and $57(64.77 \%)$ isolates, respectively recovered from human, cattle, wild bird, pig, tiger, Gecko gecko and poultry. Currently, T2544 and sopE are reannotated as pagN and sopE2, respectively.

\section{Discussion}

Salmonella infections are one of the major global public health problems. During the last decade, antimicrobial resistance and multi-drug resistance of Salmonella spp. have increased to a great extent, especially in the developing countries commensurating with increased and indiscriminate use of antimicrobial agents in the treatment of humans and animal diseases. In the present study, an attempt was made to isolate Salmonella from faecal and intestinal samples of human, animals and birds, to study serotype distribution, their antimicrobial resistance patterns, and to detect important antibiotic resistance genes and Salmonella-specific virulence genes. Out of 83 diarrhoeic stool samples collected from human, 27 (32.53\%) were positive for Salmonella. While working in the same geographical region, Borah (2017) could recover Salmonella from 45.23 per cent of diarrhoeic stool samples from man, while Purkayastha (2013) recovered the organism from 46.67 per cent of diarrhoeic human stool samples. Out of the 165 faecal samples collected from diarrhoeic cattle, 18 (10.90\%) yielded Salmonella, while Borah (2017) reported isolation of Salmonella from 6.34 per cent of bovine faecal samples. The percentage of recovery of the organism from faecal samples of diarrhoeic cattle observed in the present study was slightly higher, which might be due to the larger sample size included in the present study compared to the earlier studies reported from this part of the country. Out of 60 diarrhoeic faecal samples collected from pig, 5 (8.30\%) were positive for Salmonella. A total of $10.63 \%$ Salmonella were isolated from the rectal swabs of 94 diarrhoeic piglets (Rahman et al. 2006 ).

Out of the 302 faecal/intestinal samples collected from diarrhoeic poultry, 17 (5.63\%) yielded Salmonella. There have been reports of recovery of Salmonella from as low as 2.90 per cent (Esteban et al. 2008) and as high as $42 \%$ (Chen et al. 2020) from different parts of the world. The moderate to high prevalence of Salmonella in poultry recorded in this region might be attributed to the favourable climatic conditions like high humidity, high rainfall and to the fact that poultry is the major reservoir of Salmonella (Rahman et al, 2006). Out of the 88 isolates, 21 (23.86\%) belonged to the serovar S. enterica subsp. enterica serovar Weltevreden with the antigenic structure $3\{10\}, r, z 6 ; 22(25 \%)$ isolates belonged to serovar Enteritidis with the antigenic structure 9,12: ,m:-; 16 (18.20\%) belonged to serovar Typhi with antigenic structure 9,12,Vi,d:- and 14 (15.90\%) isolates belonged to serovar Newport with the antigenic structure 6, 8 , e, h, 1, 2, while 7 (7.95\%) isolates were found to be untypable. Salmonella Enteritidis is reported as the most common serotype worldwide (65\% of the isolates), followed by $S$. Typhimurium (12\%) (Galanis et al. 2006). This was in close agreement with the present findings. In animals, Typhimurium is the commonest serovar recovered in India followed by Weltevreden (Kumar et al. 2009). This was also in partial agreement with the present findings. 
Higher level of resistance shown by the Salmonella isolates to ampicillin, gentamicin, cefotaxime and tetracycline might be attributed to frequent and longterm use of these drugs as therapeutic agents both in man and in animals. Although chloramphenicol has long been used as one of the drugs of choice for treatment of salmonellosis in human, it was interesting to observe that lesser number of the isolates tested in the present study exhibited resistance to this drug. This might be attributed to the current trend of prescribing fluoroquinolones like ciprofloxacin more frequently in human medicine to treat cases of enteric fever. Comparatively higher percentage of isolates from cattle and poultry were found to exhibit resistance to most of the antimicrobial agents tested. It might be due to more frequent use of antimicrobial therapy in these two species compared to other species to control and prevent infectious diseases. Recently, Chloramphenicol, Ampicillin and Trimethoprim resistant $S$. Typhi that were also Ceftriaxone resistant created havoc in Pakistan and Bangladesh (Klemm et al. 2018). Here in our study also, we have recovered a few isolates showing the same resistance pattern, which is alarming. Higher level of resistance to Ampicillin and Tetracycline reported by many workers (Li et al. 2019) corroborated with the present findings. Salmonella Enteritidis causes gastroenterological disorders in human and their antimicrobial resistance is a huge concern. In the present study, Ampicillin (45.45\%), Tetracycline (54.55\%), Cefotaxime (68.18\%), Gentamicin (63.34\%) and Trimethoprim (59.09\%) resistance was recorded among S. Enteritidis isolates. Findings by AMRSN showing a nationwide downwards trend in India for resistance to Ampicillin, Chloramphenicol and trimethoprim-sulfamethaxone among $S$. Typhi (Walia et al. 2019) was in complete agreement with the present findings. Salmonella Weltevreden is found equally both in human and animals, and has importance in both as a pathogen. Wang et al. (2019) stated that antibiotic resistance behaves differently in different hosts for the same serovar. In the present study, $S$. Weltevreden showed less resistance towards Ampicillin, Ciprofloxacin, Chloramphenicol and Cephalosporins. This is indeed a good sign for the region as treatment options are still available; this was in agreement with the findings of Li et al. (2018). Salmonella Weltevreden, $S$. Typhi, S. Newport and $S$. Enteritis are the most frequently isolated serovars in this study. It was observed that Cefotaxime, a 3rd generation cephalosporin, was less effective against all these isolates. However Ceftriaxone, another 3rd generation cephalosporin, along with Cefepime, a 4th generation cephalosporin, was effective against most of the isolates, irrespective of host and serovars. Interestingly, a moderate level of resistance was shown by the isolates to Ampicillin. This may be due to the current practice of very limited use of a $\beta$ lactam antibiotics without a $\beta$ lactamase inhibitor (like Clavulinic acid) in the treatment regime for human and livestock diseases. Most of the isolates from human and cattle were resistant to Gentamicin but moderate resistance was observed in case of isolates from other host species. This may be due to the abundant use of the drug in these two species for treatment purpose. Ciprofloxacin, a fluoroquinolone, has still remained effective, as less resistance was shown by the isolates of all serovars against this drug. Isolates from Cattle, Poultry and Pig showed moderate level of resistance to Chloramphenicol, a lifesaving ICU category drug and very low resistance was exhibited by the isolates from human, particularly of serovars $S$. Weltevreden and S. Newport.

The increase in antimicrobial resistance is a threat to global health. Trimethoprim is commonly used in the treatment of urinary tract infections (UTI) in all parts of the world. However, soon after the introduction of the drug, trimethoprim resistance was reported in several species (Skold, 2001). In the present study, 41, 54 and 44 phenotypically positive Salmonella isolates which showed resistance to Ampicillin, Tetracycline and Trimethoprim, respectively were subjected to PCR for detection of resistance genes conferring resistance to these drugs. Out of 41 isolates showing resistance to ampicillin, 28 (63.64\%) showed presence of blaTEM gene, while only 2 (4.88\%) showed presence of blaOXA gene and $1(2.44 \%)$ showed presence of blaPSE gene. The presence of blaTEM in all the isolates resistant to Ampicillin as observed in the present study suggested that Ampicillin resistance in majority of Salmonella is mediated by this gene. Out of 54 isolates showing resistance to tetracycline, $9(16.67 \%)$ showed presence of tet $A$ gene and $1(1.85 \%)$ showed presence of tet $G$ gene, whereas, none of the isolates showed presence of $t e t B$ gene. This suggested involvement of some other resistance determinants in the other isolates that conferred resistance to tetracycline. Out of the 44 isolates showing resistance to trimethoprim, 5 (11.36\%) showed presence of resistance gene $d r f A 1$ but none showed presence of drfA12. Trimethoprim resistance in Salmonella is said to be mostly mediated by drfA1 gene (Brolund et al. 2010).

It has been observed that disruption of genes in Salmonella Pathogenicity Island (SPI) I of $S$. Typhimurium and $S$. Dublin blocks the secretion of Salmonella invasive proteins. The virulence genes of Salmonella spp. encoding five different Sips (Salmonella invasion protein) namely sipA, B, C, D and Eare capable of inducing apoptosis in macrophage (Kaur et al. 2012), and hence may play a vital role in Salmonella pathogenesis. Since stn has been found to localize and transcribe in juxtaposition to the gene, which encodes the dehydrogenase regulatory protein, a common and related protein among enteric micro-organisms, it is anticipated that stn determinant might be prevalent among all salmonellae (Prager et al. 1995). This gene in Salmonella is one of the chromosomally encoded genes that codes for production of enterotoxins. Observations from the present study indicated that the stn gene is universally present among all the Salmonella isolates, irrespective of the serovars, which was in agreement with Prager et al. (1995), and Murugkar et al. (2003). In the present study, sopB gene was detected in $86.36 \%$ of the Salmonella isolates which was in agreement with the findings of Rahman et al. (2006) who reported that all 50 isolates of $S$. enterica belonging to 11 serovars carried sop $B$ gene, irrespective of their serovars and source of isolation. The sefC gene was present in $51.14 \%$ of the isolates. The sef $A B C$ genes make up part of a complex sef operon responsible for the expression and assembly of SEF14 fimbriae (Clouthier et al. 1993).

\section{Conclusion}

In our study, we examined the prevalence of Salmonella and antimicrobial resistant isolates in the fecal samples of human, animals and birds. Our findings showed a high prevalence and serotype diversity in the area under study. Serovars $S$. Enteritidis, $S$. Weltevreden and $S$. Typhi were the most common serovars. Comparatively higher percentage of isolates from cattle and poultry exhibited resistance to most of the antimicrobial agents tested. It might be attributed to more frequent and rather indiscriminate use of antimicrobial agents in these two species to control and prevent infectious diseases. Moreover, the recovered Salmonella isolates exhibiting multi-drug resistance and multiple virulence genes suggested a possible risk to human and animals. Therefore, it is important to rationalize the use of antimicrobial agents to prevent vertical and horizontal transfer of MDR strains across host species. Presence of multiple virulence genes in different combinations in all the field isolates of Salmonella as revealed by the present study indicated their possible role as a pathogen in the host species. However, a molecular level phylogenetic analysis is needed to establish the inter-serovar and inter-host relationships among the Salmonella isolates.

\section{Declarations}




\section{Acknowledgements}

The authors acknowledge Department of Biotechnology (DBT), Govt of India for their financial support to carry out the entire research work.

Authors' contributions: Conceptualization- Probodh Borah; Methodology, Formal Analysis and investigation: Rupam Dutta, Iftikar Hussain; Writing - original draft preparation: Naba Kumar Deka, Dipika Malakar, Girin Hazarika; Writing - review and editing: Luit Moni Barkalita, Mridusmita Choudhury, Leena Das, Probodh Borah, Rupam Dutta; Funding acquisition: Probodh Borah; Resources: Probodh Borah; Supervision: Probodh Borah.

Funding: The study was funded by Department of Biotechnology (DBT), Govt of India.

Availability of data and material: Not applicable.

Code availability: Not applicable

Competing interests: The authors declare no competing interests.

Ethics approval: As we have collected only faecal and swab samples from different source, we took verbal consent from the individual. Tissue samples were collected from death animal by taking verbal consent from the authority.

Consent to participate: Verbal consent from the authority of each farm and individual was taken before collecting the samples.

Consent for publication: We give our consent for the publication of the submitted manuscript

\section{References}

1. Aarestrup FM, Hendriksen RS, Lockett J, Gay K, Teates K, McDer-mott PF, White DG, Hasman H, Sørensen G, Bangtrakulnonth A (2007) International spread of multidrug-resistant SalmonellaSchwarzengrund in food products. Emerg Infect Dis 13:726. 10.3201\%2Feid1305.061489

2. Abdelaziz Ed-Dra, Fouzia RF, Bouchra K, Abdellah El A, Amal A, Brahim B (2017) Prevalence, molecular and antimicrobial resistance of Salmonella isolated from sausages in Meknes, Morocco. Micro Patho 105: 340-345.

3. Borah P, Porwollik S, Desai P, Nayak P, Borah PP, Cheng P, McClelland M (2017) A simplified multiplex PCR-based typing method for common Salmonella enterica serovars supported by online server-based detection system. Indian J. Medi. Res. Vo.2(146):272-280.

4. Brolund A, Sundqvist M, Kahlmeter G, Grape M (2010) Molecular characterization of trimethoprim resistance in Escherichia coli and Klebsiella pneumonia during a two year intervention on trimethoprim use. PLoS ONE 5 (2): e9233.

5. CDC Antibiotic Resistance Threats in the United States (2019) Atlanta, GA: U.S. Department of Health and Human Services. CDC: 2019.

6. Chen Z, Bai J, Wang S, Zhang X, Zhan Z, Shen H, Zhang H, Wen J, GaoY, Liao M, Zhang J (2020) Prevalence, Antimicrobial Resistance, Virulence Genes and Genetic Diversity of Salmonella Isolated from Retail Duck Meat in Southern China. Microorganisms 8:444.

7. Choudhury M, Borah P, Sarma HK, Barkalita LM, Deka NK, Hussain IMd (2016) Multiplex-PCR assay for detection of some major virulence genes of Salmonella enterica serovars from diverse sources. Current science 111: 7.

8. Clinical and Laboratory Standards Institute (CLSI). Performance Standards for Antimicrobial Susceptibility Testing. 27th ed. CLSI supplement M100 (ISBN 1-56238) 804-5.

9. Clouthier SC, Muller Doran JL, Collinson SK, Kayl WW (1993) Characterization of three Fimbrial genes sefABC of Salmonella Enteritidis. J Bacteriol, 25232533.

10. Esteban JE, Oporto B, Aduriz G, Juste R, Hurtado A( 2008) A survey of food-borne pathogens in free-range poultry farms. Int J Food Microbiol 123:177182.

11. Galan JE, Ginocchio C, Costeas $\mathrm{P}(1992)$. Molecular and Functional Characterization of the Salmonella Invasion Gene invA: Homology of InvA to Members of a New Protein Family. J of Bacterio 174 (13) : 4338-4349.

12. Galanis E, Wong Lo Fo, D.M.A, Patrick ME, Binsztein N, Cieslik A, Chalermchikit T (2006) Web-based surveillance and global Salmonella distribution 20002002. Emerging Infect Dis 12(3): 381-388.

13. Giannella RA (1996). Salmonella In: Baron S, ed. Medical microbiology. 4th ed. Galveston (TX): University of Texas Medical Branch at Galveston.

14. Hale CR, Scallan E, Cronquist AB, Dunn J, Smith K, Robinson T, Lathrop S, Tobin-D'Angelo M, Clogher P (2012) Estimates of enteric illness attributable to contact with animals and their environments in the United States. Clin Infect Dis 54 (Suppl 5):S472-9.

15. Issenhuth-Jeanjean S, Roggentin P, Mikoleit M, Guibourdenche M, Pinna E, Nair S, Fields PI, Weill F( 2014) Supplement 2008-2010 (no. 48 ) to the WhiteeKauffmanneLe Minor scheme. Rese in Microbiol 165: 526-530.

16. Kaur J, Jain SK (2012) Role of antigens and virulence factors of Salmonella enterica serovar Typhi in its pathogenesis Microbiol Res 167: $199-210$.

17. Klemm EJ, Shakoor S, Page AJ, Qamar FN, Judge K, Saeed DK, Wong VK, Dallman TJ, Nair S, Baker S, Shaheen G, Qureshi S, Yousafzai MT, Saleem MK, Hasan Z, Dougan G, Hasan R (2018) Emergence of an extensively drugresistant Salmonella enterica serovar Typhi clone harboring a promiscuous plasmid encoding resistance to fluoroquinolones and third-generation cephalosporins. M Bio 9:e00105-18.

18. Kumar Y, Sharma A, Sehgal R, Kumar S (2009) Distribution trends of Salmonella serovars in India (2001-2005) Trans R Soc Trop Med Hyg 103:390-394.

19. Li B, Yang X, Tan H, Ke B, He D, Wang H, Chen Q, Ke C, Zhang Y (2018) Whole genome sequencing analysis of Salmonella enterica serovar Weltevreden isolated from human stool and contaminated food samples collected from the Southern coastal area of China. Int J of Food Microbi. 266: $317-323$.

Page 13/15 
20. Li Q, Yin J, Li Z, Li Z, Du Y, Guo W, Bellefeur M, Wang S, Shi H (2019) Serotype distribution, antimicrobial susceptibility, antimicrobial resistance genes and virulence genes of Salmonella isolated from a pig slaughterhouse in Yangzhou, China. AMB Expr 9:210.

21. Li R, Lai J, Wang Y, Liu S, Li Y, Liu K, Shen J, Wu C (2013) Prevalence and characterization of Salmonella species isolated from pigs, ducks and chickens in Sichuan Province, China. Intern J of Food Microbiol 163: 14-18.

22. Murugkar HV, Rahman H, Dutta PK (2003) Distribution of virulence genes in Salmonella serovars isolated from man \& animals. Indian J Med Res 117: 6670.

23. Prager R, Fruth A, Tsachape H (1995) Salmonella enterotoxin (stn) gene is prevalent among strains of Salmonella enterica, but not among Salmonella bongori and other Enterobacteriaceae. FEMS Immunol Med Microbiol 12: 47-50.

24. Prioritization of pathogens to guide discovery, research and development of new antibiotics for drug-resistant bacterial infections, including tuberculosis. Geneva: World Health Organization; 2017(WHO/EMP/IAU/2017.12). (Licence: CC BY-NC-SA 3.0 IGO).

25. Purkayastha A, Borah P, Sharma RK, Borah PP (2015) Multiplex PCR for detection of virulence gene profiles of Salmonella isolates from animals and man. Indian J. Appl. Res. 2015;5(12):142-144.

26. Rahman H (2006) Prevalence \& phenotypic expression of sopB gene among clinical isolates of Salmonella enterica. Indian J Med Res 117: 83-88.

27. Rahman H, Barman NN, Patgiri GP, Kalita N (1997) Outbreak of salmonellosis in broiler flocks in Assam. Indian J Comp Microbiol Immunol Infect Dis 18:56-58.

28. Rajkhowa S, Neher, S Pegu SR and Sarma DK (2018) Bacterial Diseases of Pigs in India: A Review. Indian J. Comp. Microbiol. Immunol. Infect. Dis. Vol. 39 (Special Issue), 2018: 29-37.

29. Skold O (2001) Resistance to trimethoprim and sulfonamides. Vet Res 32: 261-273.

30. Tanmoy AM, Westeel E., De Bruyne, K, Goris J, Rajoharison A, Sajib MSI, Van Belkum A, Saha SK, Komurian-Pradel F, Endtz HP (2018) Salmonella enterica serovar Typhi in Bangladesh: exploration of genomic diversity and antimicrobial resistance. M Bio 9:e02112-18.

31. Voetsch AC, Van Gilder TJ, Angulo FJ, Farley MM, Shallow S, Marcus R, Cieslak PR, Deneen VC, Tauxe RV( 2004) FoodNet estimate of the burden ofillness caused by nontyphoidal Salmonella infections in the United States. Clin Infect Dis 38 (Suppl. 3): S127-S134.

32. Walia K, Madhumathi J, Veeraraghavan B, Chakrabarti A, Kapil A, Ray P, Singh H, Sistla H, Ohri VC (2019) Establishing Antimicrobial Resistance Surveillance \& Research Network in India: Journey so far. Indian J Med Res 149: 164-179.

33. Wang X, Biswas S, Paudyal N, Pan H, Li X, Fang W, Yue M (2019) Antibiotic Resistance in Salmonella Typhimurium Isolates Recovered From the Food Chain Through National Antimicrobial Resistance Monitoring System Between 1996 and 2016. Front. Microbiol 10:985.

\section{Figures}

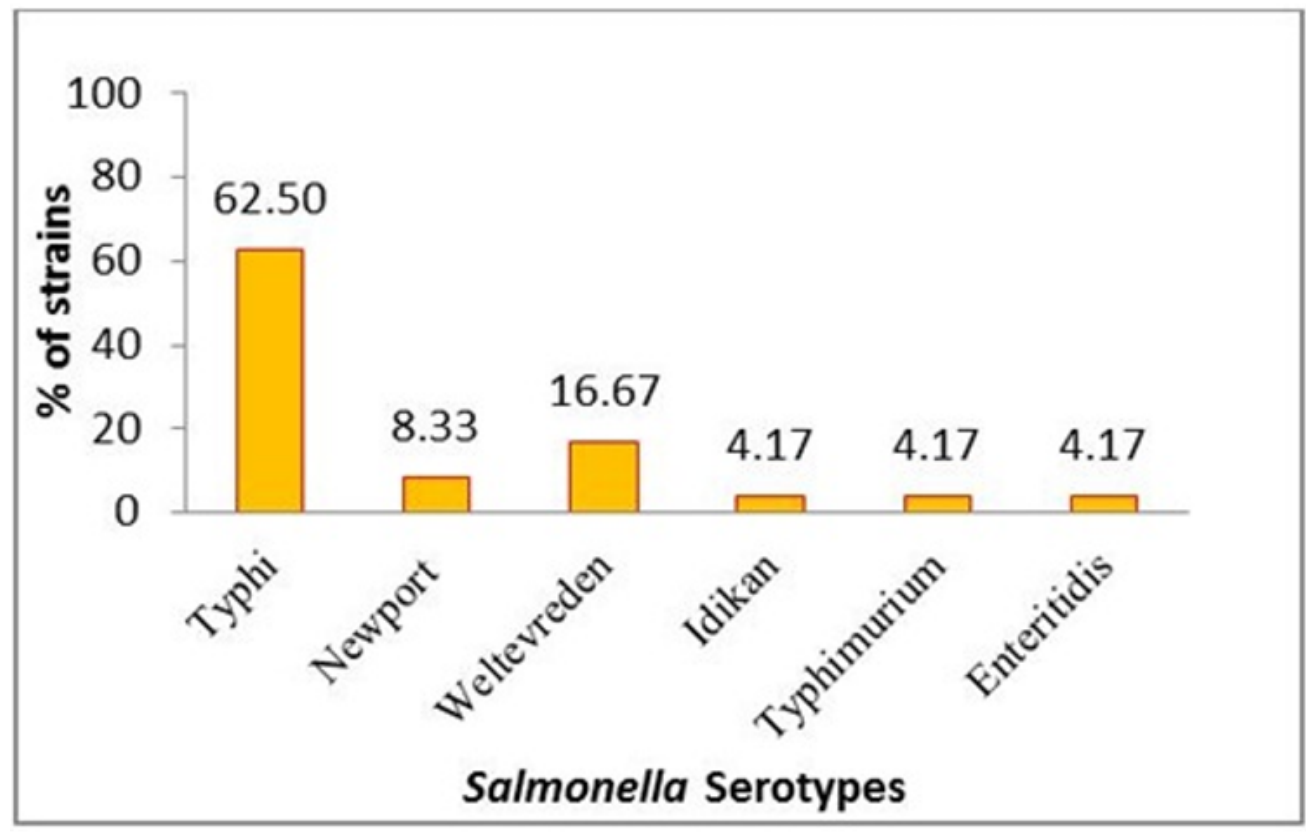

\section{Figure 1}

Frequency of isolation of different serovars of Salmonella from human 


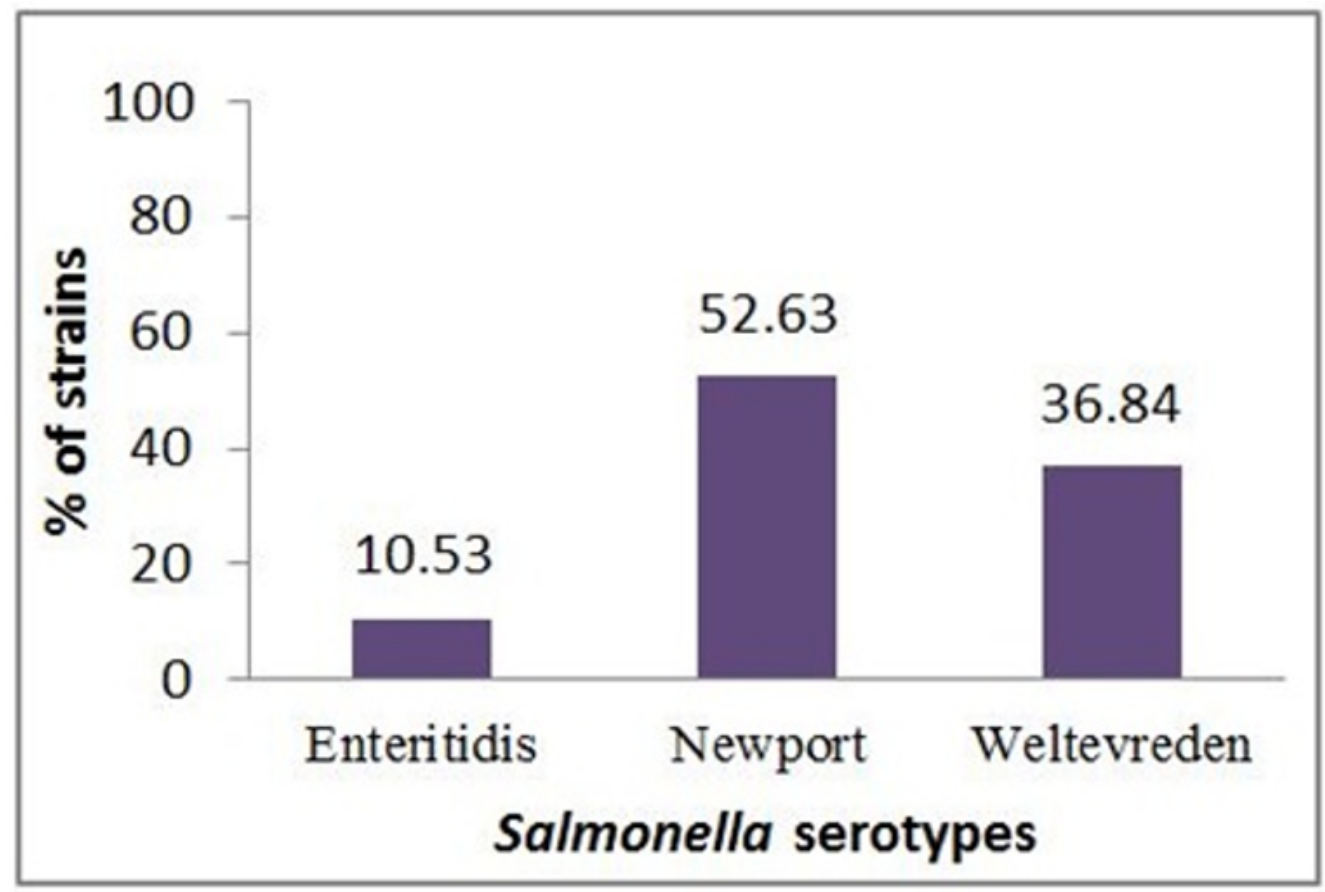

Figure 2

Frequency of isolation of different serovars of Salmonella from cattle

A

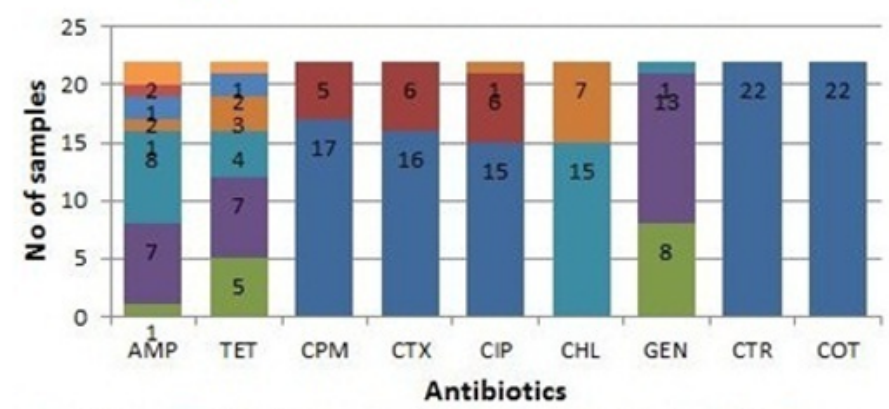

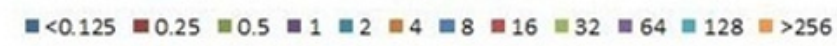

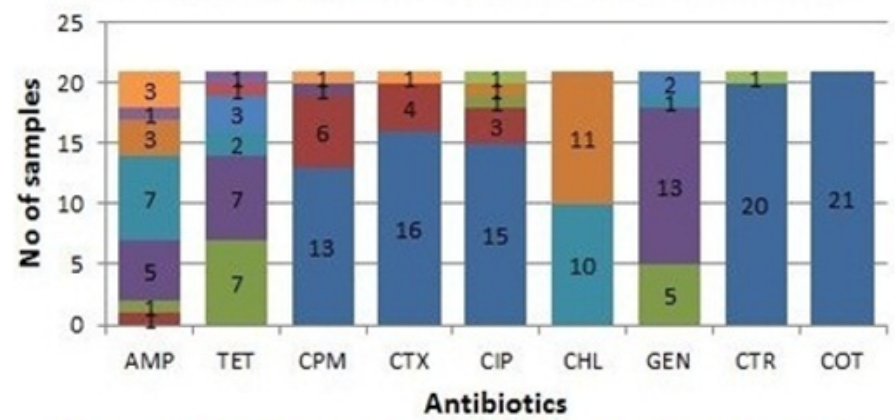

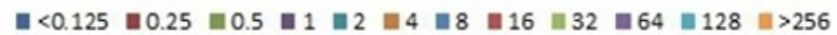

C

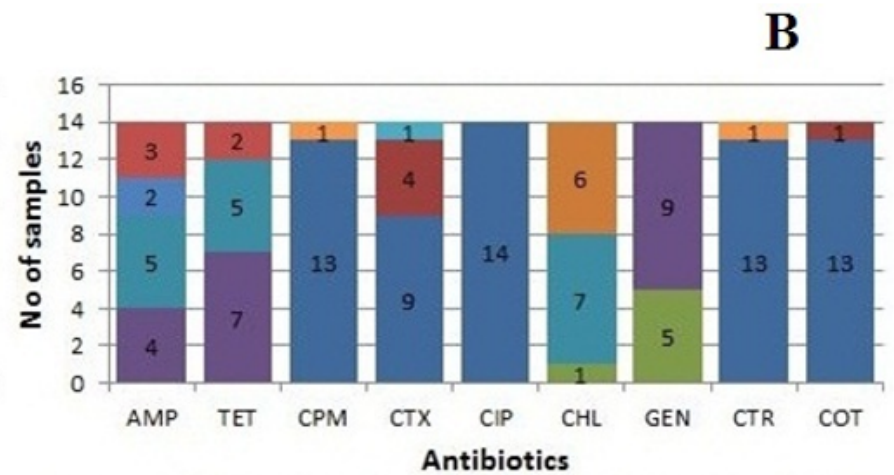

$=<0.125=0.25=0.5=1=2=4=8=16=32=64=128=>256$

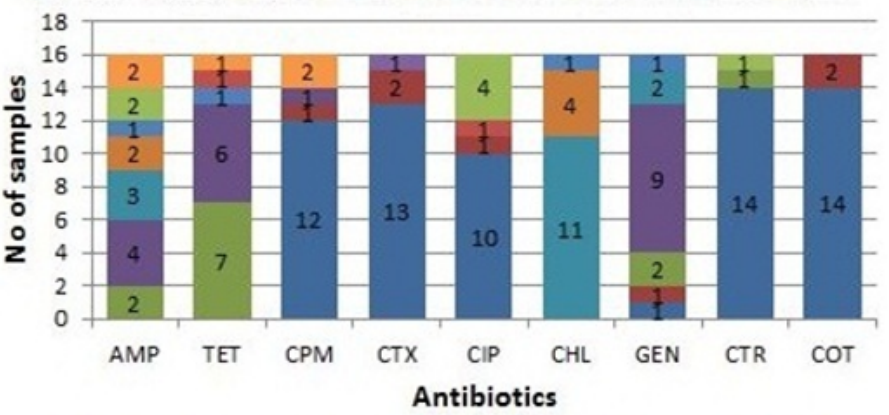

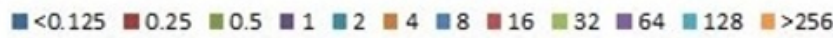

D

Figure 3

MIC data of (A) Salmonella Enteritidis, (B) Salmonella Newport, (C) Salmonella Weltevreden (D) Salmonella Typhi 